\title{
Persistent suicide risk in clinically improved schizophrenia patients: challenge of the suicidal dimension
}

\author{
Amresh Shrivastava' \\ Megan E Johnston ${ }^{2}$ \\ Nilesh Shah ${ }^{3}$ \\ Marco Innamorati ${ }^{4}$ \\ Larry Stitt ${ }^{5}$ \\ Meghana Thakar ${ }^{3}$ \\ David Lester ${ }^{6}$ \\ Maurizio Pompili4,7
}

'Silver Mind Hospital and Mental Health Foundation of India, Mumbai, India; ${ }^{2}$ Department of Psychology, University of Toronto, Toronto, ON, Canada; ${ }^{3}$ Lokmanya Tilak Municipal General Hospital, University of Mumbai, India; ${ }^{4}$ Department of Neurosciences, Mental Health and Sensory Functions, Suicide Prevention Center, Sant'Andrea Hospital, Sapienza University of Rome, Rome, Italy; ${ }^{5}$ Department of Biostatistics, The University of Western Ontario, London, ON, Canada; ${ }^{6}$ The Richard Stockton College of New Jersey, Pomona, NJ, USA; ${ }^{7}$ McLean Hospital, Harvard Medical School, Boston, MA, USA
This article was published in the following Dove Press journal:

Neuropsychiatric Disease and Treatment

2I September 2010

Number of times this article has been viewed

\begin{abstract}
Background: Suicide is a major problem in schizophrenia, estimated to affect $9 \%-13 \%$ of patients. About $25 \%$ of schizophrenic patients make at least one suicide attempt in their lifetime. Current outcome measures do not address this problem, even though it affects quality of life and patient safety. The aim of this study was to assess suicidality in long-term clinically improved schizophrenia patients who were treated in a nongovernmental psychiatric treatment centre in Mumbai, India.
\end{abstract}

Method: Participants were 61 patients out of 200 consecutive hospitalized first-episode patients with schizophrenia diagnosed according to the Diagnostic and Statistical Manual of Mental Disorders who were much improved on the Clinical Global Impression Scale-Improvement (CGI-I) scale at the endpoint of a 10-year follow-up. Clinical assessment tools included the Positive and Negative Syndrome Scale for Schizophrenia, CGI-I, Global Assessment of Functioning, and suicidality.

Results: Many of the patients, although clinically improved, experienced emerging suicidality during the 10-year follow-up period. All of the patients reported significant suicidality (ie, suicide attempts, suicidal crises, or suicidal ideation) at the end of the study, whereas only $83 \%$ had reported previous significant suicidality at baseline. No sociodemographic and clinical variables at baseline were predictive of suicidal status at the end of the 10-year follow-up.

Conclusion: Schizophrenia is a complex neurobehavioral disorder that appears to be closely associated with suicidal behavior. Adequate assessment and management of suicidality needs to be a continual process, even in patients who respond well to treatment.

Keywords: schizophrenia, suicide risk, prevention

\section{Introduction}

Suicide is a major cause of premature death among patients suffering from schizophrenia. ${ }^{1,2}$ Miles reviewed 34 studies of suicide among schizophrenia patients and estimated that $10 \%$ of them complete suicide. ${ }^{3}$ Follow-up studies have estimated that $10 \%-13 \%$ of individuals with schizophrenia die by suicide, which is the main cause of death among these patients. ${ }^{4}$ However, a recent meta-analysis estimated that only about $5 \%$ of schizophrenia patients commit suicide, ${ }^{5}$ a percentage which surprised many researchers because it was lower than previously thought. Regardless, it is still an unacceptably high rate. Inskip et al performed a meta-analysis on suicide among patients with affective disorder, alcoholism, and schizophrenia, and estimated that the lifetime risk of suicide was $6 \%$ for affective disorder, $7 \%$ for alcohol dependence, and $4 \%$ for schizophrenia ${ }^{6}$ an estimate for schizophrenia that is consistent with the estimate of Palmer et al..$^{5}$ Following an index suicide attempt, mortality from suicide
Correspondence: Maurizio Pompili Department of Psychiatry, Sant'Andrea Hospital, Sapienza University of Rome, 1035 Via di Grottarossa, 00189 Roma, Italy

Tel +39063 3775675

Fax +390633775342

Email maurizio.pompili@uniromal.it 
in schizophrenia patients may be as high as $1 \%$ per year for the next five years. ${ }^{7,8}$ Pompili et al reviewed the literature on suicide among inpatients with schizophrenia and found that the suicide rate in schizophrenia patients followed up after the first hospitalization for periods ranging from 1 to 26 years was $6.8 \% .^{9}$

Harris and Barraclough included 28 studies in their metaanalysis and found that the risk of suicide among patients diagnosed with schizophrenia exceeded that in the general population by more than eight-fold (standardized mortality rate [SMR] 8.45, confidence interval 7.98-8.95). ${ }^{10}$ Brown found that schizophrenia was associated with excess death from both natural causes (such as respiratory diseases) and unnatural causes (accidents, suicide, and homicide). ${ }^{11}$ Suicide accounted for $12 \%$ of all deaths among schizophrenia patients and about $28 \%$ of all excess deaths. According to Brown, the excess mortality was highest in first-episode or early-illness phase patients, indicating a high rate of suicide early in the illness. Danish studies that assessed SMRs in successive national cohorts suggest that the SMR may be rising in first-episode schizophrenia patients in Denmark and falling in chronic schizophrenia patients. ${ }^{12,13}$

At the same time, other data indicate that suicide risk may be elevated across the entire course of schizophrenia. A recent examination of suicides in all patients with schizophrenia in Finland over a 12-month period found that one-third of suicides occurred in patients over the age of 45 years. ${ }^{14}$ Despite great efforts, using both drug treatments and psychosocial strategies, the number of suicides among schizophrenia patients has remained unchanged, ${ }^{15}$ although Nordentoft et al have shown that suicide among Danish patients with schizophrenia has fallen, paralleling the reduction of suicide in the general population. ${ }^{16}$

The stress-diathesis suicide-risk model is helpful for understanding suicidal behavior and its management in schizophrenia. ${ }^{17}$ In this model, suicide risk factors may be either distal or proximal. Distal risk factors create a predisposing diathesis and determine the patient's response to a stressor. They include developmental, personality, biologic, and genetic variables. They affect the threshold for suicide, and increase the risk of suicide in schizophrenia when the patient experiences a proximal risk factor. Proximal (ie, trigger) factors are more closely related to suicidal behavior and act as precipitants. They include life events and, relevant to schizophrenia patients, the stress of acute episodes of mental illness, such as when relapsing into psychosis. Suicidal schizophrenia patients differ from nonsuicidal patients in distal risk factors (such as childhood trauma), and their risk of suicidal behavior may be increased by proximal risk factors, such as an exacerbation of illness caused by treatment noncompliance, adverse life events, or comorbid substance abuse and depression, as well as sleep problems. ${ }^{18}$

The present paper examines the presence of suicidality in a cohort of clinically improved patients with first-episode schizophrenia followed up for 10 years. The objective was to assess whether, with continuous treatment, suicidal thoughts, plans, and attempts were present in patients who showed satisfactory clinical improvement.

\section{Methods}

\section{Study site}

This study was conducted in a nongovernmental psychiatric treatment center (licensed under the Indian Mental Health Act, 1987) in Mumbai, India. Ethics permission was obtained from the local independent research ethics board. Appropriate consents were received from all patients.

\section{Sample and study design}

Two hundred consecutive, hospitalized, first-episode patients entered a long-term follow-up study. Almost half (49\%) of the patients were lost during the 10-year follow-up which, although high, appears to be a general pattern in patients with early psychosis. ${ }^{13}$ Patients available at the endpoint of 10 years and rated as "improved" and "much improved" $(n=61)$, as measured by the Clinical Global Impression Scale-Improvement (CGI-I), were recruited for the present study to assess the presence of suicidal behavior. Forty-three (73\%) of the patients were male, and the mean duration of illness prior to treatment was 14.0 months, with a standard deviation of \pm 8.0.

To assess suicide risk, we used a measurement scale of 1 to 5 ( 1 being the lowest value and 5 being the highest). This semistructured scale has been tested in local conditions and used in other studies. ${ }^{19}$ We compared the characteristics of the patients and treatment variables at baseline and at follow-up.

\section{Measures}

Clinical assessment of patients was conducted using the Positive and Negative Syndrome Scale for Schizophrenia (PANSS), ${ }^{20} \mathrm{CGI},{ }^{21}$ and Global Assessment of Functioning (GAF). ${ }^{22}$ The CGI was developed for use in clinical trials sponsored by the National Institutes of Mental Health to provide a brief, stand-alone assessment of the clinician's view of the patient's global functioning prior to and after initiating a study medication. ${ }^{21}$ The scale provides an overall clinician-determined summary measure that takes into account all available information, including knowledge of 
the patient's history, psychosocial circumstances, symptoms, behavior, and impact of symptoms on the patient's ability to function. The CGI comprises two dimensions, ie, severity of psychopathology (CGI-S) and improvement after initiation of treatment (CGI-I). Both dimensions are rated on a seven-point Likert-type scale (from 1 "very much improved" to 7 "very much worse since the initiation of treatment" for the CGI-I, and from 1 "normal" to 7 "among the most extremely ill patients" for the CGI-S).

Suicidality was rated by clinical interviews and a review of medical files. Subjects were categorized in one of the following classes:

- Attempted suicide - a potentially self-injurious behavior, associated with at least some intent to die as a result of the act; evidence that the individual intended to kill himself/ herself, at least to some degree, can be explicit or inferred from the behavior or circumstance; a suicide attempt may or may not result in actual injury

- Experiencing a suicidal crisis - preparatory acts toward imminent suicidal behavior; the individual takes steps to injure himself or herself but is stopped by self or others from starting the self-injurious act before the potential for harm has started

- Contemplating suicide - active thoughts about killing oneself, not accompanied by preparatory behavior

- Occasional death wishes - passive thoughts about wanting to be dead

- No suicidal intent - an outcome free from suicidality defined as the absence of any suicidal behavior or ideation, or occasional presence of death wish.

Fifty-five percent of patients were on monotherapy, 25\% were on two or more antipsychotics, including a combination of two atypicals in $18 \%$ of cases. Percent usage pattern and daily mean dosage (available for about $88 \%$ of the patients) was 23\% (295 mg) for clozapine, 7.4\% (5.3 mg) for risperidone, $7.6 \%$ (17.6 mg) for olanzapine, $10.4 \%$ (558 mg) for quetiapine, $23.8 \%(16 \mathrm{mg}$ ) for aripiprazole, and $5.7 \%$ (112 mg) for ziprasidone.

\section{Statistical methods}

We used signed rank tests for comparisons between baseline versus the end of the study, and one-way analyses of variance and Chi-square tests $\left(\chi^{2}\right)$ for comparisons between independent groups (patients who attempted suicide during the study, those who experienced suicidal crisis, and those who had suicidal ideation). Because a higher number of analyses may increase Type I errors (the probability of accepting the alternative hypothesis when it is not true), we corrected our results with a Bonferroni correction for multiple testing. The data were analyzed using SAS version 9.1 (SAS Institute, Cary, NC). Probability values less than 0.05 were considered to be statistically significant.

\section{Results}

Table 1 lists the baseline characteristics of the sample of clinically improved schizophrenia patients who had moderate to severe symptomatology at admission (PANSS $108.5 \pm 14.2$; positive symptoms $29.6 \pm 5.2$; negative symptoms $20.9 \pm 6.4$ ) and severe limitation of social and work functioning (GAF $48.0 \pm 10.9$ ).

About $83 \%$ of patients reported a previous suicide attempt, suicidal crisis, or suicidal ideation ( $24 \%$ reported previous suicide attempts, $26 \%$ reported experience of a suicidal crisis, and $33 \%$ reported suicidal ideation). At the end of the study, $100 \%$ of patients reported a suicide attempt, suicidal crisis, or suicidal ideation during the course of the study (see Table 2). Thus, despite the fact that all patients were assessed as improved or much improved on the CGI-I between the baseline evaluation and the end of the study, suicidality increased significantly $(P<0.001)$. About $72 \%$ of patients had attempted suicide or experienced a suicidal crisis since the first hospitalization, whereas only $50 \%$ of patients reported having attempted suicide or experiencing a suicidal crisis before the first hospitalization. This figure indicates that many schizophrenia patients who are considered to be clinically improved experience emerging suicidality during treatment. Because most patients experienced emerging suicidality (suicidal behavior or ideation), a prior suicide attempt was not a good predictor of suicidality during the treatment phase. No sociodemographic and clinical variables at baseline were associated with suicidal status at the end of the study (see Table 3). PANSS was significant only before correcting for multiple testing.

Table I Baseline characteristics of schizophrenia patients $(n=6 I)$

\begin{tabular}{ll}
\hline Characteristic & $\begin{array}{l}\text { Mean (SD) or } \\
\text { frequency (\%) }\end{array}$ \\
\hline Age at intake (years) & $31.8(7.6)$ \\
Duration of illness (months) & $14.0(8.0)$ \\
Men & $43(70.5 \%)$ \\
PANSS total & $108.5(14.2)$ \\
PANSS PS & $29.6(5.2)$ \\
PANSS NS & $20.9(6.4)$ \\
PANSS disorganization (>3) & $52(86.7 \%)$ \\
CGI-S & $5.6(1.0)$ \\
GAF & $48.0(10.9)$ \\
\hline
\end{tabular}

Abbreviations: PANSS, Positive and Negative Syndrome Scale for Schizophrenia; CGI-S, Clinical Global Impression Scale-Improvement-Severity; NS, negative symptoms; PS, positive symptoms; GAF, Global Assessment of Functioning; SD, standard deviation. 
Table 2 Suicidality at baseline and the end of the study

\begin{tabular}{lll}
\hline Suicidality & Baseline & End of study \\
\hline Attempted suicide & $15(24.1 \%)$ & $30(48.3 \%)$ \\
Suicidal crisis & $16(25.9 \%)$ & $14(23.3 \%)$ \\
Suicidal ideation & $20(32.8 \%)$ & $17(28.3 \%)$ \\
Death wish & $7(12.1 \%)$ & $0(0.0 \%)$ \\
None & $3(5.2 \%)$ & $0(0.0 \%)$ \\
\hline
\end{tabular}

Notes: For difference between baseline and end of study, $P<0.00$ I, signed rank test.

Furthermore, neither untreated illness duration before first hospitalization (less versus more than six months) nor age at intake $(<24$ years versus $>25$ years) was associated with subsequent suicidality (not reported in the tables).

Thus, baseline severity of symptomatology, positive and negative symptoms, and global functioning in daily life (as well as gender, age, and duration of untreated illness before first hospitalization) may not help clinicians predict suicidality in schizophrenia patients. The mean positive symptoms score was reduced by more than $65 \%$ between baseline and the end of the study. Negative symptoms were also reduced, but to a much lesser degree than were positive symptoms. It was observed that $23 \%-25 \%$ of patients showed social recovery on two or three parameters (life functioning, employability, interpersonal functioning, and ability to live independently). Basically, clinical symptoms improved but, in terms of social outcome, improvements were not as robust.

\section{Discussion}

The aim of this study was to assess suicide risk in first-episode schizophrenia patients during a 10-year follow-up period. Surprisingly, patients who improved in the psychopathologic components of their disorder were at high risk of suicide. One of the main issues in contemporary psychiatry is the fact that treating schizophrenia does not necessarily mean that proper suicide prevention is taken into account.

The lack of association between suicide and psychiatric disorders has been documented in many studies. ${ }^{23,24}$ The conclusion derived from psychological autopsy studies, namely that the vast majority of individuals who die by suicide suffered from a mental disorder at the time of their death, has been shown to suffer from several biases. ${ }^{25}$ First, scholars worldwide use the term psychological autopsy for any retrospective investigation. Such studies lack comprehensive data from interview of key persons. It is rather easy to classify a person as depressed when in fact he or she was understandably sad over the state of his or her life. Most of the data obtained in psychological autopsy studies are derived from a forensic examination, physician, or death register, and much less often from family members or friends who could make sense of the depressive features which are distinguishable from clinical depression. Suicide is a problem of the human condition or, as Shneidman points out “ ... a dissatisfaction of the status quo". ${ }^{26,27}$

Scholars have come to believe that alternative theoretical models must be found, because the vast majority of depressed, schizophrenic, alcoholic, or organically psychotic patients do not attempt or complete suicide. ${ }^{28-30}$ Much suicide research focuses on psychiatric patients, but ignores the cry for help that results from loss, humiliation, failure, and shame. ${ }^{26}$

Therefore, one of the errors in suicide prevention is the assumption that treating a psychiatric disorder will also reduce suicide risk. The evidence supports instead the

Table 3 Association between baseline characteristics and subsequent suicidality

\begin{tabular}{|c|c|c|c|c|c|}
\hline & $\begin{array}{l}\text { Attempted } \\
\text { suicide } \\
(\mathbf{n}=30)\end{array}$ & $\begin{array}{l}\text { Suicidal crisis } \\
(n=14)\end{array}$ & $\begin{array}{l}\text { Suicidal } \\
\text { ideation } \\
(n=17)\end{array}$ & Test statistic & $P$ value \\
\hline $\begin{array}{l}\text { Age at intake } \\
\text { (years) }\end{array}$ & $32.5(7.0)$ & $31.6(9.8)$ & $30.6(6.6)$ & $F(2,57)=0.34$ & 0.72 \\
\hline $\begin{array}{l}\text { Duration of illness } \\
\text { (months) }\end{array}$ & $13.9(8.1)$ & I3.9 (7.5) & I4.2 (8.9) & $F(2,57)=0.01$ & 0.99 \\
\hline Male gender & I8 (60.0\%) & 10 (7I.4\%) & I 4 (82.4\%) & $\chi^{2}(\mathrm{df}=2)=1.6 \mathrm{I}$ & 0.45 \\
\hline PANSS & $106.5(14.3)$ & I03.4 (II.8) & II $5.8(14.1)$ & $F(2,57)=3.67$ & 0.03 \\
\hline PS & $28.6(6.0)$ & $30.5(3.9)$ & $30.8(4.6)$ & $F(2,57)=1.19$ & 0.31 \\
\hline NS & $21.7(6.6)$ & $21.7(5.6)$ & $19.2(6.5)$ & $F(2,57)=0.94$ & 0.40 \\
\hline $\begin{array}{l}\text { Abnormal } \\
\text { disorganization }(>3)\end{array}$ & $25(83.3 \%)$ & 10 (7I.4\%) & $16(94.1 \%)$ & $\chi^{2}(\mathrm{df}=2)=|.5|$ & 0.47 \\
\hline CGI-S at baseline & $5.6(1.0)$ & $5.6(1.0)$ & $5.5(0.8)$ & $F(2,57)=0.07$ & 0.94 \\
\hline GAF & $49.4(9.6)$ & $50.8(11.4)$ & 43.3 (II.6) & $F(2,57)=2.4 I$ & 0.10 \\
\hline
\end{tabular}

Notes: Bonferroni corrected $P$ value: $0.05 / 11$ l $=0.005$.

Abbreviations: PANSS, Positive and Negative Syndrome Scale for Schizophrenia; CGI-S, Clinical Global Impression Scale-Improvement-Severity; NS, negative symptoms; PS, positive symptoms; GAF, Global Assessment of Functioning. 
notion that suicide prevention requires more than psychiatric treatment. ${ }^{23,24,31}$ The results of the present study indicate that clinical improvement is not a key element in decreasing suicide risk. On the contrary, improvement in schizophrenia symptoms may increase suicidality. Given the fact that improvement of the psychosis may lead to awareness of the illness, suicide risk may be the result of painful insight into one's own impairment. ${ }^{32}$

Suicide prevention requires specific inquiry which must involve careful attention to suicidal risk and protective factors, as well as exploring warning signs for suicide, such as talking about suicide and death and having no reason to live. Most suicidal individuals give definite warnings of their suicidal intention, but significant others are either unaware of the significance of these warnings or do not know how to respond to them. Suicidal individuals may withdraw from friends and social activities and may have a recent severe loss (especially a relationship) or a threat of a significant loss. Moreover, they may show drastic changes in behavior and lose interest in hobbies, work, and school, as well as increasing their use of alcohol or drugs and showing unwillingness to "connect" with potential helpers.

Hopelessness has been reported to be a more important indicator than depression of suicide risk. Studies using the Beck Hopelessness Scale have found that the extent of negative attitudes about the future (pessimism) was a better predictor of suicidal intent than depression. ${ }^{33,34}$

Drake et al have pointed to the need for empathic support in reducing suicide risk. ${ }^{35}$ These authors suggested that clinicians should acknowledge the patient's despair, discuss losses and daily difficulties, and help the patient establish new and accessible goals.

Behavior of medical staff is a potential cause of suicide when staff members fail to recognize suicide risk because of personal problems or difficulties with the topic of suicide. Pompili et al pointed to the role of nurses in the prevention of suicide in schizophrenia. ${ }^{36,37}$ These authors outlined the many difficulties in the care of a schizophrenic patient who is at risk of suicide, and drew attention to staff "countertransference" reactions to these patients. A relevant concept is "terminal malignant alienation". ${ }^{38,39}$ Some patients, particularly those with recurrent relapses and resistance to treatment, may be perceived by staff as manipulative, provocative, unreasonable, overdependent, and feigning disability. ${ }^{38-40}$ Patients with fluctuating suicidal ideation are particularly likely to fall into these categories, and this may lead to underreporting of suicidal ideation by nursing staff. This may result in a critical attitude toward patients and a lower level of support, leading to alienation. The combination of such alienation and fluctuating suicidal ideation can lead to failure to recognize the seriousness of suicide risk..$^{38,39}$

A proper treatment regimen is of paramount importance for reducing suicide risk among schizophrenia patients. Clozapine is associated with the strongest antisuicidal effect when compared with other pharmacologic agents. ${ }^{41}$ Electroconvulsive treatment, lithium therapy, and psychosocial interventions are also of great importance for reducing suicide risk. ${ }^{1}$

The present study has a number of limitations, including a small sample size, lack of a widely used instrument to assess suicide risk, and the cross-sectional nature of the study in a naturalistic clinical setting. These limitations impair generalization of the results. Lastly, we did not analyze exacerbations of illness during the 10-year treatment, which may be associated with emergent suicidality. However, our analysis points to the absence of long-term predictors for suicide risk when assessing the clinical status of schizophrenia patients.

Despite these caveats, our study points to a core problem in dealing with schizophrenia patients. Clinicians should be aware of the unique nature of suicidality which accompanies the psychiatric disorder, but which may be independent of the disorder. Specific inquiry into a patient's suicide risk is required in order to initiate measures to prevent suicide during the treatment of schizophrenia. Suicide might be better understood as phenomenon-centered in the individual, ie, the motives for suicide can be traced to the variables surrounding the individual viewed as a unique human being whose personality contains the real reasons for wishing suicide..$^{42,43}$

\section{Disclosure}

The authors report no conflicts of interest in this work.

\section{References}

1. Pompili M, Lester D, Innamorati M, Tatarelli R, Girardi P. Assessment and treatment of suicide risk in schizophrenia. Expert Rev Neurother. 2008;8(1):51-74.

2. Roy A, Pompili M. Management of schizophrenia with suicide risk. Psychiatr Clin North Am. 2009;32(4):863-883.

3. Miles CP. Conditions predisposing to suicide: A review. J Nerv Ment Dis. 1977;164(4):231-246.

4. Caldwell CB, Gottesman II. Schizophrenia - a high-risk factor for suicide: Clues to risk reduction. Suicide Life Threat Behav. 1992;22(4):479-493.

5. Palmer BA, Pankratz VS, Bostwick JM. The lifetime risk of suicide in schizophrenia: A reexamination. Arch Gen Psychiatry. 2005;62(3):247-253.

6. Inskip HM, Harris EC, Barraclough B. Lifetime risk of suicide for affective disorder, alcoholism and schizophrenia. Br J Psychiatry. 1998; 172:35-37.

7. Ettlinger R. Evaluation of suicide prevention after attempted suicide. Acta Psychiatr Scand Suppl. 1975;260:1-135. 
8. Meltzer HY. Suicidality in schizophrenia: A review of the evidence for risk factors and treatment options. Curr Psychiatry Rep. 2002;4(4):279-283.

9. Pompili M, Mancinelli I, Ruberto A, Kotzalidis GD, Girardi P, Tatarelli R. Where schizophrenic patients commit suicide: A review of suicide among inpatients and former inpatients. Int $J$ Psychiatry Med. 2005;35(2):171-190.

10. Harris EC, Barraclough B. Suicide as an outcome for mental disorders. A meta-analysis. Br J Psychiatry. 1997;170:205-228.

11. Brown S. Excess mortality of schizophrenia. A meta-analysis. Br J Psychiatry. 1997;171:502-508.

12. Munk-Jorgensen P, Mortensen PB. Incidence and other aspects of the epidemiology of schizophrenia in Denmark, 1971-87. Br J Psychiatry. 1992;161:489-495.

13. Licht RW, Mortensen PB, Gouliaev G, Lund J. Mortality in Danish psychiatric long-stay patients, 1972-1982. Acta Psychiatr Scand. 1993;87(5):336-341.

14. Heila H, Isometsa ET, Henriksson MM, Heikkinen ME, Marttunen MJ, Lonnqvist JK. Suicide and schizophrenia: A nationwide psychological autopsy study on age- and sex-specific clinical characteristics of 92 suicide victims with schizophrenia. Am J Psychiatry. 1997;154(9): 1235-1242.

15. Meltzer HY, Conley RR, de Leo D, et al. Intervention strategies for suicidality. J Clin Psychiatry Audiograph. 2003;6:1-16.

16. Nordentoft M, Laursen TM, Agerbo E, Qin P, Hoyer EH, Mortensen PB. Change in suicide rates for patients with schizophrenia in Denmark, 1981-97: Nested case-control study. BMJ. 2004;329(7460):261.

17. Mann JJ, Waternaux C, Haas GL, Malone KM. Toward a clinical model of suicidal behavior in psychiatric patients. Am J Psychiatry. 1999; 156(2):181-189.

18. Pompili M, Lester D, Grispini A, et al. Psychiatry Res. 2009;167(3): 251-257.

19. Shrivastava A, Gopa S. Comparative study of risperidone and haloperidol on clinical and psychosocial parameters in treatment of schizophrenia: A randomised open trial. Ind J Psychiatry. 2000;42(1):52-56.

20. Kay SR, Fiszbein A, Opler LA. The positive and negative syndrome scale (PANSS) for schizophrenia. Schizophr Bull. 1987;13(2):261-276.

21. Guy W. ECDEU Assessment Manual for Psychopharmacology. Washington, DC: US Department of Health, Education, and Welfare; 1976.

22. American Psychiatric Association. Diagnostic and Statistical Manual of Mental Disorders, 4th ed. Washington, DC: American Psychiatric Association; 1994.

23. Bertolote JM, Fleischmann A, de Leo D, Wasserman D. Psychiatric diagnoses and suicide: Revisiting the evidence. Crisis. 2004; 25(4):147-155.

24. de Leo D. Suicide prevention is far more than a psychiatric business. World Psychiatry. 2004;3(3):155-156.
25. Pouliot L, de Leo D. Critical issues in psychological autopsy studies. Suicide Life Threat Behav. 2006;36(5):491-510.

26. Shneidman ES. Final contribution to suicidology. In: Pompili M, editor. Suicide in the Words of Suicidologists. New York, NY: Nova Science Publishers Inc; In press.

27. Shneidman ES. How I read. Suicide Life Threat Behav. 2005; 35(2):117-120.

28. Lester D. Suicide as a Learned Behavior. Springfield, IL: Charles Thomas; 1987.

29. Lester D. Questions and Answers about Suicide. Philadelphia, PA: The Charles Press; 1989.

30. Leenaars A. Psychotherapy with Suicidal People. Chichester, UK: John Wiley; 2004.

31. Bertolote JM, Fleischmann A, de Leo D, Wasserman D. Suicide and mental disorders: Do we know enough? Br J Psychiatry. 2003;183: 382-383.

32. Pompili M, Ruberto A, Kotzalidis GD, Girardi P, Tatarelli R. Suicide and awareness of illness in schizophrenia: An overview. Bull Menninger Clin. 2004;68(4):297-318.

33. Beck AT, Weissman A, Lester D, Trexler L. The measurement of pessimism: The hopelessness scale. J Consult Clin Psychol. 1974;42(6):861-865.

34. Beck AT, Steer RA. Beck Hopelessness Scale-Manual. San Antonio, TX: Harcourt Brace Javanovich, Inc.; 1988.

35. Drake RE, Bartels SJ, Torrey WC. Suicide in schizophrenia: Clinical approaches. In: Williams R, Dalby JT, editors. Depression in Schizophrenia. New York, NY: Plenum Press; 1989:153-169.

36. Pompili M, Mancinelli I, Girardi P, Tatarelli R. Nursing schizophrenic patients who are at risk of suicide. J Psychiatr Ment Health Nurs. 2003;10(5):622-624.

37. Pompili M, Mancinelli I, Girardi P, Tatarelli R. Making sense of nurses' role in the prevention of suicide in schizophrenia. Issues Ment Health Nurs. 2004;25(1):5-7.

38. Morgan HG, Priest P. Assessment of suicide risk in psychiatric inpatients. Br J Psychiatry. 1984;145:467-469.

39. Morgan HG, Priest P. Suicide and other unexpected deaths among psychiatric in-patients. The Bristol confidential inquiry. Br J Psychiatry. 1991;158:368-374.

40. Schwartz DA, Flinn DE, Slawson PF. Suicide in the psychiatric hospital. Am J Psychiatry. 1975;132(2):150-153.

41. Meltzer HY. Evidence-based treatment for reducing suicide risk in schizophrenia. In: Pompili M, Tatarelli R, editors. Evidence-based Practice in Suicidology: A Sourcebook. Göttingen, Germany: Hogrefe; 2010

42. Pompili M. Exploring the phenomenology of suicide. Suicide Life Threat Behav. 2010;40(3):234-244.

43. Pompili M. From bench to bedside in the prevention of suicide: A never ending back and forth journey. Crisis. 2010;31(2):59-61.
Neuropsychiatric Disease and Treatment

\section{Publish your work in this journal}

Neuropsychiatric Disease and Treatment is an international, peerreviewed journal of clinical therapeutics and pharmacology focusing on concise rapid reporting of clinical or pre-clinical studies on a range of neuropsychiatric and neurological disorders. This journal is indexed on PubMed Central, the 'PsycINFO' database and CAS, and is the official

\section{Dovepress}

journal of The International Neuropsychiatric Association (INA). The manuscript management system is completely online and includes a very quick and fair peer-review system, which is all easy to use. Visit $\mathrm{http}: / /$ www.dovepress.com/testimonials.php to read real quotes from published authors. 